\title{
Transient Lupus Anticoagulant and False-Positive Antibody Tests for Cytomegalovirus in Epste in-Barr Virus Infectious Mononucleosis
}

\author{
Bicakci $\mathrm{Z}^{1 *}$ and Bozkurt $\mathrm{BH}^{2}$ \\ ${ }^{1}$ Department of Pediatric Hematology and Oncology, \\ Kafkas University, Turkey \\ ${ }^{2}$ Department of Pediatrics, Kafkas University, Turkey \\ *Correspondling author: Zafer Bicakci, Department of \\ Pediatric Hematology and Oncology, Kafkas University, \\ Faculty of Medicine, Kars, Turkey
}

Received: December 03, 2018; Accepted: J anuary 21, 2019; Published: J anuary 28, 2019

\begin{abstract}
Infectious mononucleosis may be associated with Epstein-Barr Virus (EBV), as well as the Cytomegalovirus (CMV), and may lead to the development of Lupus Anticoagulant (LA) or anticardiolipin antibodies. CMV proteins may also react with IgM antibodies against EBV during the Enzyme-Linked Immunosorbent Assay (ELISA) test. It can be difficult to differentiate the infections caused by either virus as they lead a very similar clinical course with similar laboratory test results. In this study, we present a three-year-old girl with EBV-induced infectious mononucleosis. The laboratory tests indicated the presence of transient lupus anticoagulant (prolonged aPTT) and resulted in a false positivity for cytomegalovirus antibody. She had hepatosplenomegaly, elevation of transaminases, atypical lymphocytosis (78\%), prolonged Partial Thromboplastin Time (aPTT), and the laboratory test for LA was positive. The laboratory tests to detect viruses showed CMV IgM (+), CMV PCR (-), EBV VCA $\operatorname{IgM}(+)$, and EBV VCA IgG (+). About a month after the onset of the disease, elevation of the transaminases and aPTT prolongation returned to normal, and hepatosplenomegaly was resolved. Approximately five months after the onset of the disease, lupus anticoagulant and CMV IgM were not positive. No complications such as thrombosis or bleeding were observed. In conclusion; polyclonal B lymphocyte activation in EBV infectious mononucleosis may result in the development of transient lupus anticoagulant (prolonged aPTT) and also false-positive antibody tests for cytomegalovirus due to the antigenic similarity between these two viruses.
\end{abstract}

Keywords: Epstein-Barr virus; Infectious mononucleosis; Lupus anticoagulant; Cytomegalovirus

\section{Introduction}

Epstein-Barr Virus (EBV) and Cytomegalovirus (CMV), the members of the herpes virus family, are the most common viruses that cause infectious Mononucleosis (EM) characterized by fever, pharyngitis, and lymphadenopathy. At least $90 \%$ of the world's population is infected with EBV/CMV. The disease may transform into a latent form after the primary infection and may reactivate after many years in conditions associated with immunodeficiencies. A primary infection may also occur shortly in infancy after the elimination of the maternal antibodies. EBV is the most common cause of infectious mononucleosis in childhood. Primary CMV infections account for $7 \%$ of mononucleosis syndromes, manifesting symptoms so similar to the EBV-induced mononucleosis that it is almost impossible to make a differential diagnosis [1].

It is known that viral infections cause the emergence of antiphospholipid antibodies (aPLs) in both adults and children [2]. aPLs are frequently transient, especially in children after viral infections [3]. The levels of aPLs have been reported to be as high as $30-60 \%$ during acute Epstein-Barr Virus (EBV) infections [4]. A transient lupus anticoagulant may be detected due to polyclonal B-lymphocyte activation induced by the EBV infection [5].
Circulating Lupus Anticoagulants (LA), together with AntiCardiolipin (aCL) antibodies, form a subset of the heterogeneous autoantibody group called antiphospholipid antibodies (aPL). These antibodies develop against phospholipid-dependent plasma proteins, mainly prothrombin or $\beta 2$-glycoprotein $\mathrm{I}$, and inhibit certain in vitro phospholipid-dependent coagulation reactions (typically the conversion of prothrombin to thrombin), leading to prolonged activated Partial Thromboplastin Time (aPTT). aPLs have also been reported in association with several clinical conditions including viral infections, autoimmune disorders, and malignancies; as well as in healthy-looking individuals (primary antiphospholipid syndrome) [2].

LA or aCL antibodies may occur in both EBV and CMV infections [2]. The clinical manifestations of a cytomegalovirus-associated mononucleosis-like syndrome share many clinical features with those of classical EBV-induced infectious mononucleosis. Besides the similar haematological and hepatic findings in infections induced by both of these viruses, a primary EBV infection may also induce the emergence of a cross-reaction with low titres of CMV-IgM [6].

In this case report, we aimed to present a three-year-old female patient with EBV-induced infectious mononucleosis, in whom transient lupus anticoagulant (prolonged aPTT) and false-positive 
antibody tests for cytomegalovirus were detected.

\section{Case Report}

A three-year-old girl had been admitted to another hospital for four days due to high fever, fatigue, tonsillitis, and oedema in the eyelids. The laboratory findings included a leukocyte count of $8500 \times 10^{9} / \mathrm{L}$ and a platelet count of $192 \times 10^{9} / \mathrm{L}$. The levels of haemoglobin and haematocrit were $12.5 \mathrm{~g} / \mathrm{dL}$ and $37 \%$, respectively. The following laboratory values included AST $85 \mathrm{U} / \mathrm{L}$, ALT $98 \mathrm{U} / \mathrm{L}$, Total bilirubin (T.bil) $0.57 \mathrm{mg} / \mathrm{dL}$, and Direct Bilirubin (D.bil) 0.28 $\mathrm{mg} / \mathrm{dL}$. The urinalysis was negative for protein. After the evaluation of the clinical and laboratory findings, the patient was diagnosed with tonsillitis. As the complaints of the patient continued, she was admitted to our hospital one day later with the following findings including mild oedema in the eyelids, swelling of the tonsils, tonsilar crypt exudate, hepatomegaly (extending $2 \mathrm{~cm}$ inferiorly under the last rib on the right), and splenomegaly (extending $1 \mathrm{~cm}$ under the last rib on the left). Atypical lymphocytes were detected in the peripheral smear and elevated levels of transaminases were present. Infectious mononucleosis was considered in the diagnosis. The following results were detected including CMV IgG (+), CMV IgM (+), CMV PCR (-), EBV VCA IgM (+), and EBV VCA IgG (+). Routine coagulation tests showed a prolonged aPTT, however, fibrinogen and D-dimer levels were normal. The prolonged aPTT level was detected in a 1: 1 dilution of the patient's plasma with normal human plasma. A mixing assay did not improve aPTT, suggesting that anticoagulants were present in the circulation excluding a coagulation factor deficiency. The levels of the coagulation factors, which might have potentially affected aPTT (VIII, IX, XI, and XII) were also normal. Tests for intrinsic coagulation factor inhibitors were negative. Lupus anticoagulant was $112.6 \mathrm{sec}$ $(<44)$, lupus screening was $112.6 \mathrm{sec}(<44)$, lupus verification was 57.8 $\sec (<37)$, and lupus screening/verification was $2.52(<1.3)$. For this reason, negative results were obtained for the anticardiolipin $\operatorname{IgM}$, anticardiolipin IgG, anti-phosphatidylserine IgM, antiphospholipid IgG, anti-dsDNA, and ANA 6.5. The tests for C3, C4 IgA, IgG, and IgM revealed normal results. The results of the serological tests for hepatitis A, B, and C; HIV, and TORCH were negative. Therefore, LA diagnosis criteria were met [7]. About a month after the onset of the disease, hepatosplenomegaly disappeared, and the elevated levels of transaminases and the prolonged aPTT were normalised. Approximately five months after the onset of the disease, the positive results of the lupus anticoagulant and CMV IgM tests disappeared. No complications such as thrombosis or bleeding were observed. The patient was considered to have a temporarily positive test result for lupus anticoagulant secondary to EBV infection (Table 1).

\section{Discussion}

Although Infectious Mononucleosis (IM) has initially been described in adults, it is an acute febrile disease also of adolescents, children, and even infants, involving the lymphatic system. More than $90 \%$ of the IM syndrome is reported to be caused by Epstein-Barr Virus (EBV), followed by CMV and Toxoplasma gondii, accounting for approximately $5-7 \%$ and less than $1 \%$ of the cases, respectively [6].

EBV infection occurs when the virus envelope/helical glycoprotein (gp) 350/220 binds to the C3d complement receptor (CR2 or CD21) of the B lymphocytes. Three viral protein (gH-gL-gp42) complexes are required to enter into the B lymphocytes. Class II Human Leukocyte
Table 1: Selected laboratory values at baseline and in the fifth month of followup.

\begin{tabular}{|c|c|c|}
\hline & $\begin{array}{l}\text { At the time of } \\
\text { admission }\end{array}$ & $\begin{array}{l}\text { fifth month of } \\
\text { follow-up }\end{array}$ \\
\hline Leukocyte count $\left(\times 10^{9} / \mathrm{l}\right)$ & 8.7 & 5.6 \\
\hline Neutrophil count $\left(\times 10^{9} / \mathrm{l}\right)$ & 1.43 & 1.7 \\
\hline Lymphocyte count $\left(\times 10^{9} / \mathrm{l}\right)$ & 6.35 & 3.51 \\
\hline Atypical lymphocyte count (\%) & 78 & 20 \\
\hline Haemoglobin (g/dl) & 12 & 12.4 \\
\hline Thrombocyte count $\left(\times 10^{9} / \mathrm{l}\right)$ & 199 & 332 \\
\hline CRP (mg/dL) & 2.2 & 0.06 \\
\hline AST (U/l) & 62 & 12 \\
\hline $\operatorname{ALT}(\mathrm{U} / \mathrm{l})$ & 85 & 38 \\
\hline LDH (U/l) & 445 & 263 \\
\hline PT (s) & 11.2 & 11.2 \\
\hline PT-INR & 0.86 & 0.86 \\
\hline aPTT (s) (control 33.7 s) & 81 & 28.2 \\
\hline $\begin{array}{l}\text { Lupus anticoagulant scanning (LA1) } \\
(<44 \mathrm{~s})\end{array}$ & 112.6 & 44.1 \\
\hline $\begin{array}{l}\text { Lupus anticoagulant correction (LA2) } \\
(<37 \mathrm{~s})\end{array}$ & 57.8 & 39.9 \\
\hline LA1:LA2 dRVVT (s) (<1.3) & 2.52 & 1.1 \\
\hline EBV VCA IgM (+) (1.1>negative) & 2.3 & 2.56 \\
\hline EBV VCA IgG (+) (1.1>negative) & 2.64 & 3.34 \\
\hline Anti CMV IgM (+) (1.1> negative) & 2.01 & 0.56 \\
\hline Anti CMV IgG (+) (10> negative) & 200 & 51.4 \\
\hline CMV PCR & $(-)$ & \\
\hline
\end{tabular}

CRP: C-Reactive Protein; AST: Aspartate Aminotransferase; ALT: Alanin Aminotransferase; LDH: Lactate Dehydrogenase; PT: Prothrombin Time; PTINR: Prothrombin Time-International Normalized Ratio; aPTT: activated Partial Thromboplastin Time; EBV VCA: Epstein-Barr Virus Viral Capsid Antigens; CMV: Cytomegalovirus; PCR: Polymerase Chain Reaction

Antigen (HLA) molecules serve as a chimeric for gp42. Neutralizing antibodies occurring after the primary infection are specific to EBV, allowing for making the diagnosis of an EBV infection. Although the neutralising antibodies against EBV are involved in preventing the spread of EBV to extra B cells, the control of an EBV infection is achieved by the cellular immunity involving the CD8+ T-cells. NK and CD4+ T-cells also play a role in the control of EBV infection. However, it is the primary task of the memorial cytotoxic CD8+ T-cells (EBV-CTLs) to control an EBV infection. Initially, EBV-CTL cells are responsible for the emergence of the majority of infectious mononucleosis-specific atypical lymphocytes (Downey cells), as well as resulting the emergence of other cells that cause lymphocytosis [8].

Many clinical and laboratory findings are common in infectious mononucleosis caused by both EBV and CMV [6]. In patients presenting with clinical findings raising a suspicion of an EBV infection, lymphocytosis should be detected at a minimum level of more than $50 \%$ (more than $10 \%$ atypical lymphocytes) [9]. The diagnosis is confirmed by the serological tests showing positive results for EBV VCA IgM (+) and EBV VCA IgG (+), as well as by the presence of hepatosplenomegaly, elevated levels of transaminases, lymphocytosis $\left(6.35 \times 10^{9} / 1\right)$, atypical lymphocytes (Downey cells), CMV IgG (+), CMV IgM (+), and CMV PCR (-). The clinical findings 
and laboratory data of our patient were consistent with the literature.

Earlier studies reported that coinfection (double infection) might produce intense reactions in $\mathrm{CD} 8+\mathrm{T}$-cells, and might increase immunosuppression and hypercytokinemia, increasing the risk of serious complications [10]. In our patient, immunosuppression or hypercytokinemia did not occur, and no serious complications developed. Therefore, a coinfection was not considered in the diagnosis. About a month after the onset of the disease, hepatosplenomegaly disappeared, and the elevated levels of the transaminases and aPTT prolongation were normalised. After about five months following the onset of the disease, CMV PCR was negative, and lupus anticoagulant and CMV IgM were not positive. This condition was accepted as a transient lupus anticoagulant and false-positive antibody test for cytomegalovirus. A previous study confirmed our diagnosis in the sense that approximately $40 \%$ of the patients with acute EBV infection were shown to have positive IgM antibody titers against CMV [6]. In vitro studies have shown that CMV proteins used in ELISA as recombinant antigens can react with the increased quantities of IgM antibodies produced against other herpes viruses. Epstein-Barr Nuclear Antigen 1 (EBNA-1) contains repeating Gly-Ala sequences as an important antigenic determinant for IgM antibody production during an acute infection. The antigen construct of EBV and CMV was shown to have a repeated homology in the N-terminal half of EBNA-1 with glycine-rich motifs in CMV polypeptides and Gly-Ala sequences [11]. This antigenic similarity may be the cause of falsepositive antibody test results for cytomegalovirus.

Two main types of LA have been reported in children. The first type is often a benign and transient type; often seen in very young children and associated with infections. The second type is associated with autoimmune diseases, persisting for a long time and is strongly associated with thromboembolic complications [2]. In our patient, no clotting factor deficiencies were detected, and no thrombosis or bleeding episodes were observed. Our patient was considered to develop the first type of LA due to the prolongation of aPTT, the presence of transient lupus anticoagulant, and a false positive antibody test for cytomegalovirus in EBV infectious mononucleosis.

Lupus anticoagulant is an acquired immunoglobulin (usually IgG, sometimes IgM or IgA) that binds negatively charged calcium and phospholipid components of factor Xa and Va. The presence of circulating LA is suggested by the detection of prolongation in the coagulation tests such as phospholipid-dependent aPTT (which does not return to normal when mixed with normal plasma) [12]. Anti-cardiolipin IgM, anti-cardiolipin IgG, anti-phospholipid IgG, anti-phosphatidylserine IgM, ANA, and anti-dsDNA were found to be negative in our patient. Based on the criteria of the International Society of Thrombosis and Haemostasis [7], the diagnosis of our patient was confirmed by demonstrating the presence of the prolonged aPTT, the positive result obtained in the mixing assay, the positive result for LA, and by the lupus screening/verification rate.

Lupus anticoagulant does not usually cause a bleeding disorder. The main reason for looking for the lupus anticoagulant in assays is to avoid intensive examination and treatment before surgery. If this antibody (Lupus anticoagulant) is detected in a young child with an acute viral infection, the test must be repeated to follow-up the disappearance of the antibody within a few months [12]. In our patient, lupus anticoagulant became negative in five months after the disease onset, and lupus screening/validation rate was found to be $1.10(<1.3)$.

Even though there are often similarities between anticardiolipin or anti- $\beta 2$-glycoprotein I antibodies and LA, these antibodies are not all the same. In general, LA is more specific but anticardiolipin antibodies are more sensitive to detect an antiphospholipid antibody syndrome [12]. In our patient, anti-cardiolipin antibodies were negative but the lupus anticoagulant was positive because of the antiphospholipid antibody syndrome.

\section{Conclusion}

In the EBV-associated infectious mononucleosis, transient lupus anticoagulant (prolonged aPTT) and a false-positive antibody test for cytomegalovirus may coexist as a result of polyclonal B lymphocyte activation.

\section{References}

1. Wang $X$, Yang K, Wei C, Huang Y, Zhao D. Coinfection with EBV/CMV and other respiratory agents in children with suspected infectious mononucleosis. Virol J. 2010; 7: 247.

2. Shiomou K, Galanakis E, Tzoufi M, Tsaousi C, Papadopoulou ZL. Transient lupus anticoagulant and prolonged activated partial thromboplastin time secondary to Epstein-Barr virus infection. Scand J Infect Dis. 2002; 34: 67-69.

3. Mizumoto H, Maihara T, Hiejima E, Shiota M, Hata A, Seto S, et al. Transient antiphospholipid antibodies associated with acute infections in children: a report of three cases and a review of the literature. Eur J Pediatr. 2006; 165: 484-488.

4. Ben-ChetritE, Wiener-Well Y, Fadeela A, Wolf DG. Antiphospholipid antibodies during infectious mononucleosis and their long term clinicalsignificance. J Clin Virol. 2013; 56: 312-315.

5. Yamazaki M, Asakura $\mathrm{H}$, Kawamura $\mathrm{Y}$, Ohka $\mathrm{T}$, Endo $\mathrm{M}$, Matsuda $\mathrm{T}$. Transient lupus anticoagulant induced by Epstein-Barr virus infection. Blood Coagul Fibrinolysis. 1991; 2: 771-774.

6. Medović R, Igrutinović Z, Radojević-Marjanović R, Marković S, Rasković Z, Simović $A$, et al. Clinical and laboratory differences between Epstein-Barr and cytomegalovirus infectiousmononucleosis in children. Srp Arh Celok Lek. 2016; 144: 56-62.

7. Pengo V, Tripodi A, Reber G, Rand JH, Ortel TL, Galli M, et al. Update of the guidelines for lupus anticoagulant detection. Subcommittee on Lupus Anticoagulant/Antiphospholipid Antibody of the Scientific and Standardisation Committee of the International Society on Thrombosis and Haemostasis. J Thromb Haemost. 2009; 7: 1737-1740.

8. Gross TG. Infectious mononucleosis and other Epstein-Barr virusrelated disorders. In: Greer JP, Arber DA, Glader B, List AF, Means Jr, RT,Paraskevas F, Rodgers GM, editors. Wintrobe's Clinical Hematology, $13^{\text {th }}$ ed. Philadelphia: Lippincott Williams \&Wilkins. 2014; 1324-1341.

9. Lennon P, Crotty M, Fenton JE. Infectious mononucleosis. BMJ. 2015; 350 h1825.

10. Wakiguchi $H$, Hisakawa $H$, Kubota $H$, Kurashige $T$. Strong response of $T$ cells in infants with dual infection by Epstein-Barr virus and cytomegalovirus. Pediatr Int. 1999; 41: 484-489.

11. Lang D, Vornhagen R, Rothe M, Hinderer W, Sonneborn $\mathrm{HH}$, Plachter B. Cross-reactivity of Epstein-Barr virus-specific immunoglobulin $M$ antibodies with cytomegalovirus antigens containing glycine homopolymers. Clin Diagn Lab Immunol. 2001; 8: 747-756.

12. Acharya SS, Srangi SN. Disorders of coagulation. In: Philip Lanzkowsky, Jeffrey M. Lipton, Jonathan D. Fish, editors. Lanzkowsky's manuel of pediatric hematology and oncology, $6^{\text {th }}$ ed. India: MPS Limited, Chennai. $2016 ; 279-333$ 